\title{
Nuevas evidencias sobre el artesanado alimentario panadero en Hispania. A propósito de dos sellos localizados en Tarraco
}

\author{
New evidence for the bread-making trade of Hispania. \\ Comments on two stamps found in Tarraco
}

\author{
Josep F. Roig PÉRez \\ Institut Català d'Arqueologia Clàssica \\ Avinguda Reina Maria Cristina, 50-52, esc. B, 1r 2a, E-43002 Tarragona \\ jfroig@icac.cat
}

\author{
Macarena Bustamante-Álvarez \\ Universidad de Granada. Campus de la Cartuja \\ Calle del Prof. Clavera, s/n, E-18011 Granada \\ mbustamante@ugr.es
}

En este trabajo se presentan dos sellos de terracota recuperados en Tarraco que, por su morfología, asociamos a la producción de masas panaderas selladas. Además de un análisis iconográfico, abordamos un estudio de su contexto arqueológico no sólo desde el punto de vista cronológico, sino también intentando aportar significación a esta práctica común en Hispania.

\section{PALABRAS CLAVE}

SELLO PANADERO, PISTRINA, TARRACO, REPRESENTACIÓN ZOOMORFA, MEDUSA

In this paper two terracotta stamps discovered in Tarraco are presented. Due to their morphology, we associate both with the production and stamping of bakery dough. In addition to an analysis of their iconography, we offer an evaluation of their archaeological context, not only with regard to dating but also with respect to the significance of this common practice in Hispania.

\section{KEYWORDS}

BAKERY STAMPS, PISTRINA, TARRACO, ZOOMORPHIC REPRESENTATION, MEDUSA 


\section{Introducción}

El análisis del artesanado alimentario hispanorromano atiende a múltiples facetas que nos ayudan a desgranar la paleodieta consumida en Hispania. Sin lugar a dudas, uno de los productos alimentarios consumidos, por la idoneidad geográfica de esta región, fueron los cereales, así como sus derivados. Sin embargo, tanto la materia prima como los productos resultantes, debido a su carácter perecedero, no suelen llegar hasta nosotros, a diferencia de lo que sí pasa con otros lugares donde las características de conservación son extraordinarias, como en los enclaves vesubianos. Por esta razón, la arqueología debe atender a otras piezas de este complejo palimpsesto de la alimentación en época antigua, como sus medios de producción. En este caso, tanto las instalaciones, denominadas "pistrina", como los instrumentos utilizados en la cadena productiva son las pistas que nos ayudan a completar el panorama (para más datos sobre el tema: Salido y Bustamante, 2014).

En este estudio traemos a colación una serie de evidencias sobre el proceso de panificación localizadas en el solar de la antigua Tarraco (Tarragona), un lugar de especial significación por su condición de capitalidad de la provincia. En concreto, presentamos dos piezas inéditas en cerámica que responden a la tipología de punzón alimentario.

\section{Sobre los sellos alimentarios y la panificación}

Cuando nos referimos a los sellos de panadero hacemos alusión a unas piezas con unas características físicas muy concretas. Por regla general son discos cerámicos de unos 10-15 cm que presentan, al menos, en una de sus caras decoración en positivo o en negativo. La parte posterior aparece bien espatulada o bien modelada a mano, como se desprende de las huellas digitales que suelen quedar marcadas. En algunas ocasiones, como en uno de los ejemplares que nos compete, dichas piezas presentan un mamelón o mango para facilitar la mejor aprehensión del sello.

En cuanto al por qué se les ha asociado a productos alimentarios, además de por su aparición en contextos de panificación, se han tenido en cuenta otras variables (Salido y Bustamante, 2014: 53). En primer lugar, la ausencia de ejemplares en positivo, que hubieran esclarecido alguna idea sobre la posible generación de piezas en formato no perecedero (caso de las figuras de barro). En segundo lugar, es sintomática la reiteración de una morfología muy apta para trabajar con una mano y en un formato cercano a los panecillos de época romana (crustulae). En tercer lugar, llama la atención la reiteración de unos ciclos iconográficos bien definidos que fueron las claves para la definición de su funcionalidad. En cuarto lugar, también tenemos interesantes paralelos etnográficos que, en algunos casos, llegan hasta la actualidad. Con todo ello, la historiografía no duda de la función vinculada al sellado de productos alimentarios; sin embargo, sí podría generarse 
cierta discusión a la hora de determinar a qué producto nos referimos ya que, en cualquier momento, se podría haber usado para otra masa de manera polivalente.

Uno de los primeros trabajos que reflexionaron sobre la temática de los instrumentos vinculados a la panificación y las panaderías fue el de Pasqui (1906), en concreto en Ostia. Esta senda fue continuada por Müller (1909) o Drexel (1916), quienes de manera más directa los vincularon al sellado para actos religiosos puntuales, algo que cobra sentido si tenemos en cuenta la escasa presencia en el registro hispano.

Quizás los estudios más esclarecedores en lo que se refiere al establecimiento de una estrecha vinculación con los actos votivos fue el de Deonna (1919), al focalizar su investigación en las piezas que se encontraban en Suiza y en el limes septentrional. O bien Alföldi (1938), al asociarlo al posible sellado en momentos muy concretos, como en el caso del dies natalis del emperador o en la conmemoración de algunos momentos importantes de su vida.

En relación a la historiografía hispana, la temática comienza a ser un clásico muy recurrente vinculado al artesanado alimentario. Los primeros hallazgos en Hispania se dieron a conocer en 1921. Concretamente, nos referimos a un conjunto de gran interés localizado en Córdoba y que se asoció directamente a un complejo alimentario (De la Torre, 1921; De los Santos, 1949). También fueron significativos los hallazgos acaecidos en la región próxima de la Mauretania Tingitana (Quintero, 1942), que permitieron lanzar una nueva hipótesis de trabajo vinculada al posible origen de las piezas, ya que aportaban un componente exógeno al mundo romano, hasta el momento no valorado, que podría tener vinculación con las terracotas placa de época feno-púnica (Salido y Bustamante, 2014: 53).

A pesar de la cantidad de piezas localizadas en la península ibérica, son pocas las que aparecen en contexto. Entre los ejemplares destacamos uno de Málaga, datado entre los siglos II y IV d. C. (Pérez-Malumbres, 2018), y otro sello localizado en el año 2001 en Lucentum (Alicante), que se ha podido datar en la primera mitad del II d. C. (Salido y Bustamante, 2014: 54).

\section{Contextualización de los hallazgos}

Las dos piezas que ahora presentamos proceden del solar de la antigua capital de la Hispania Citerior, Tarraco. En concreto, la primera de ellas se localizó en una excavación practicada en el actual Mercado Central y, por consiguiente, en una zona extramuros al suroeste de la ciudad, cercana al foro de la colonia y a una de las puertas de acceso a la misma (figs. 1 y $5 a$ ).

Los resultados obtenidos en dicha excavación, acometida entre los años 2007 y 2012 , consistieron en el hallazgo de un volumen de restos de época romana con una amplia cronología, desde inicios del siglo I d. C. hasta mediados del siglo v d. C. (Brú et al., 2012: 313-323). Al primer momento cronológico (fase 1) corresponden los restos de una vía, canalizaciones para la captación o evacuación de aguas para uso agrícola o industrial, restos de encajes de cimentación de sillares, una pequeña cantera y dos potentes zonas de extrac- 


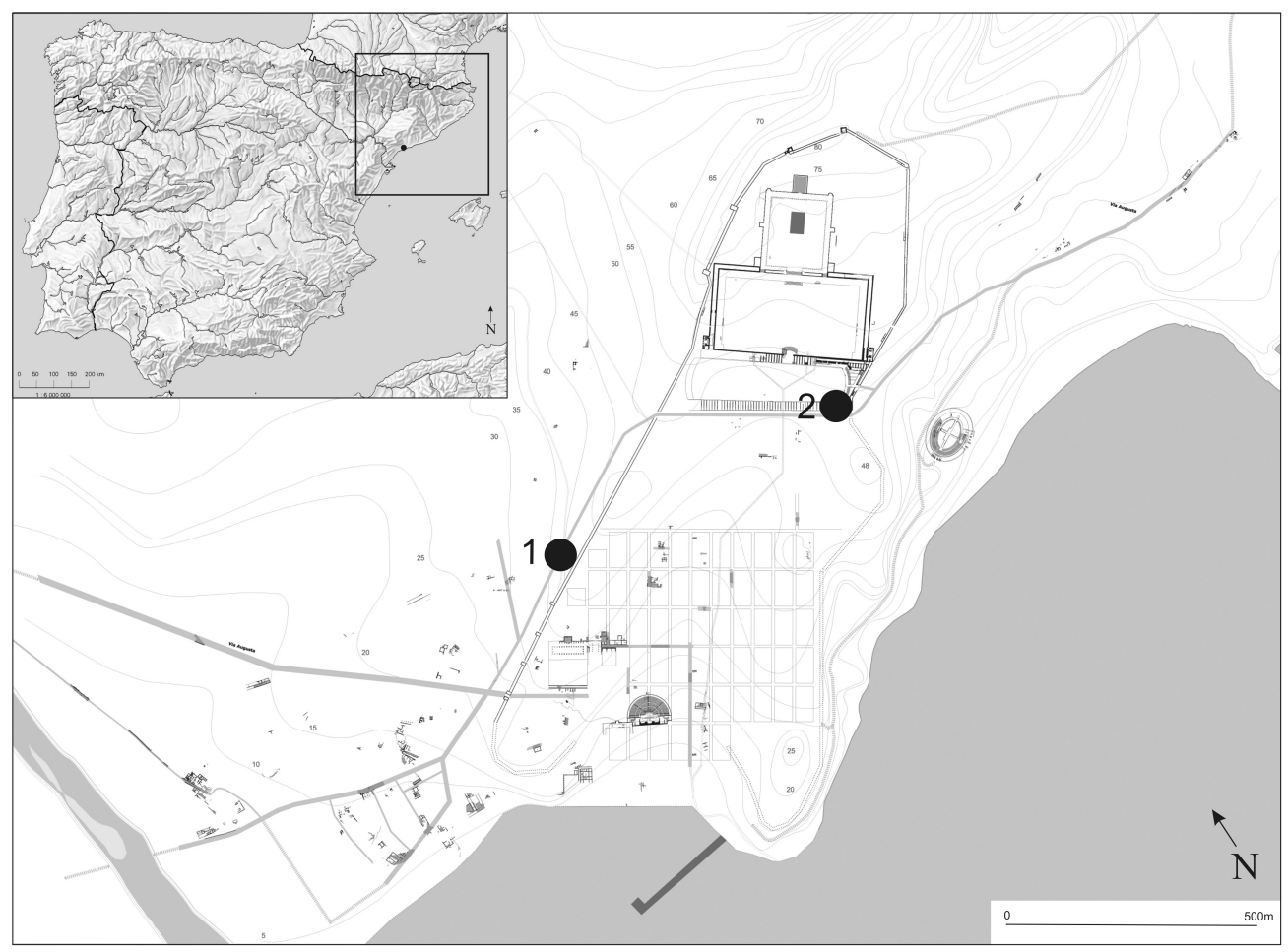

Figura 1. Parte superior izquierda, localización de Tarragona dentro de la península ibérica. Abajo, planimetría de la antigua Tarraco entre la dinastía flavia i antonina, 69-180 d. C.), con indicación de los lugares donde se localizan los solares reseñados. Punto 1: Mercado Central. Punto 2: Cabecera del circo romano. Plano base extraído de la Planimetría Arqueológica de Tarraco (Macias et al., 2007).

ción de arcilla. La fase 2 se caracteriza por la amortización de los frentes de extracción de materia primera de la fase anterior (fig. 2) y por la edificación en época de los emperadores Tiberio-Claudio (fig. 3) de una taberna lusoria (Roig y Teixell, 2015: 221-227), además de una serie de dependencias de carácter industrial (Roig, 2014: 55-74). Fue en esta fase cuando se localizó la pieza que ahora centra nuestra atención.

El momento de máxima actividad edilicia, datada a finales del siglo I d. C., corresponde a la fase 3, en la que destacan dos áreas industriales, una potente canalización de agua y parte de una domus con atrio y doble porticado. La fase 4, datada durante el siglo II d. C., se caracteriza por el embellecimiento y ampliación de la domus de la fase anterior y por la edificación de una serie de estructuras asociadas a un acceso subterráneo interpretado como un área de culto a las ninfas (Teixell et al., 2016: 115-131). Después de un periodo de destrucción e incendio datado durante la década del $260 \mathrm{~d}$. C., identificamos la fase 5 , con una datación a partir del año 276 d. C. (Teixell, 2012: 87-103) y caracterizada por la 

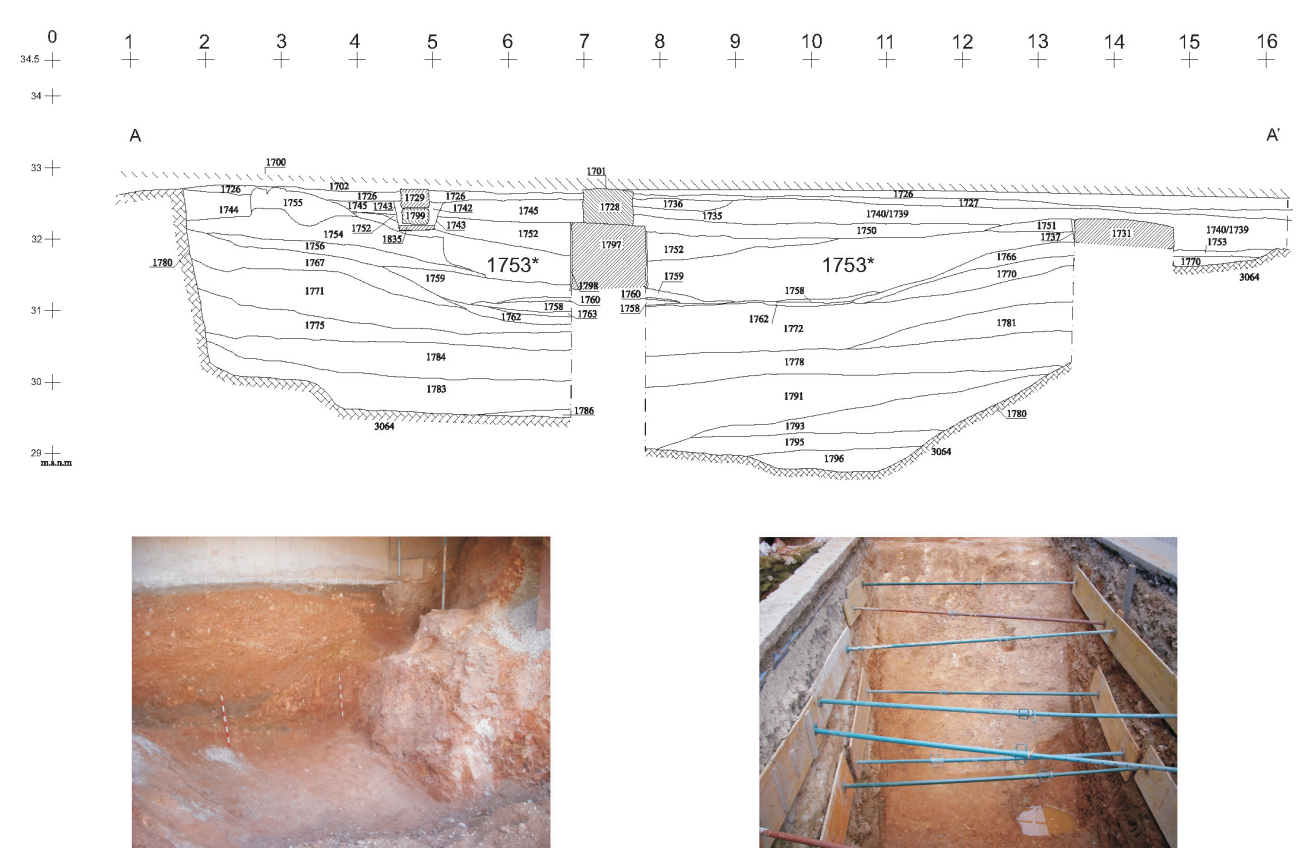

Figura 2. Arriba, sección arqueológica con indicación del estrato 1753. Abajo, imágenes del frente de extracción de arcilla una vez finalizados los trabajos arqueológicos (autor: Codex / J. F. Roig).

construcción de diferentes estructuras de carácter industrial. De esta fase son también las primeras inhumaciones. La fase número 6, datada en torno al siglo IV d. C., se caracteriza por la documentación de un vertedero y un nuevo proyecto constructivo que amplía las estructuras industriales de la fase precedente a partir del desmontaje de parte de las estancias residenciales de la domus de la fase 3. La colmatación definitiva de todo el espacio se produjo a mediados del siglo $\mathrm{v}$ d. C.

La segunda pieza (figs. 1 y 5b) procede de una serie de intervenciones desarrolladas entre los años 1977-1990 en la cabecera del circo romano. Estas excavaciones arqueológicas sacaron a la luz un gran número de estructuras arquitectónicas asociadas con el ángulo meridional de la cabecera oriental del circo romano (graderío, escaleras, bóvedas de subestructura de la fachada monumental, así como la puerta triumphalis) (fig. 4).

También se encontró un tramo de la muralla republicana, una puerta monumental asociada con la entrada oriental de la Via Augusta a la ciudad y un horreum (Dupré et al., 1988; Dupré y Subías, 1993: 603-609; Mar et al., 2015: 170-211; Díaz y Teixell, 2017: 230-237).

La pieza en cuestión fue recuperada en 1987, más concretamente en la bóveda K, en un nivel del que, desgraciadamente, no poseemos información precisa. Sólo tenemos algunos de los materiales que la acompañaron, entre los que se encuentran cerámicas de 


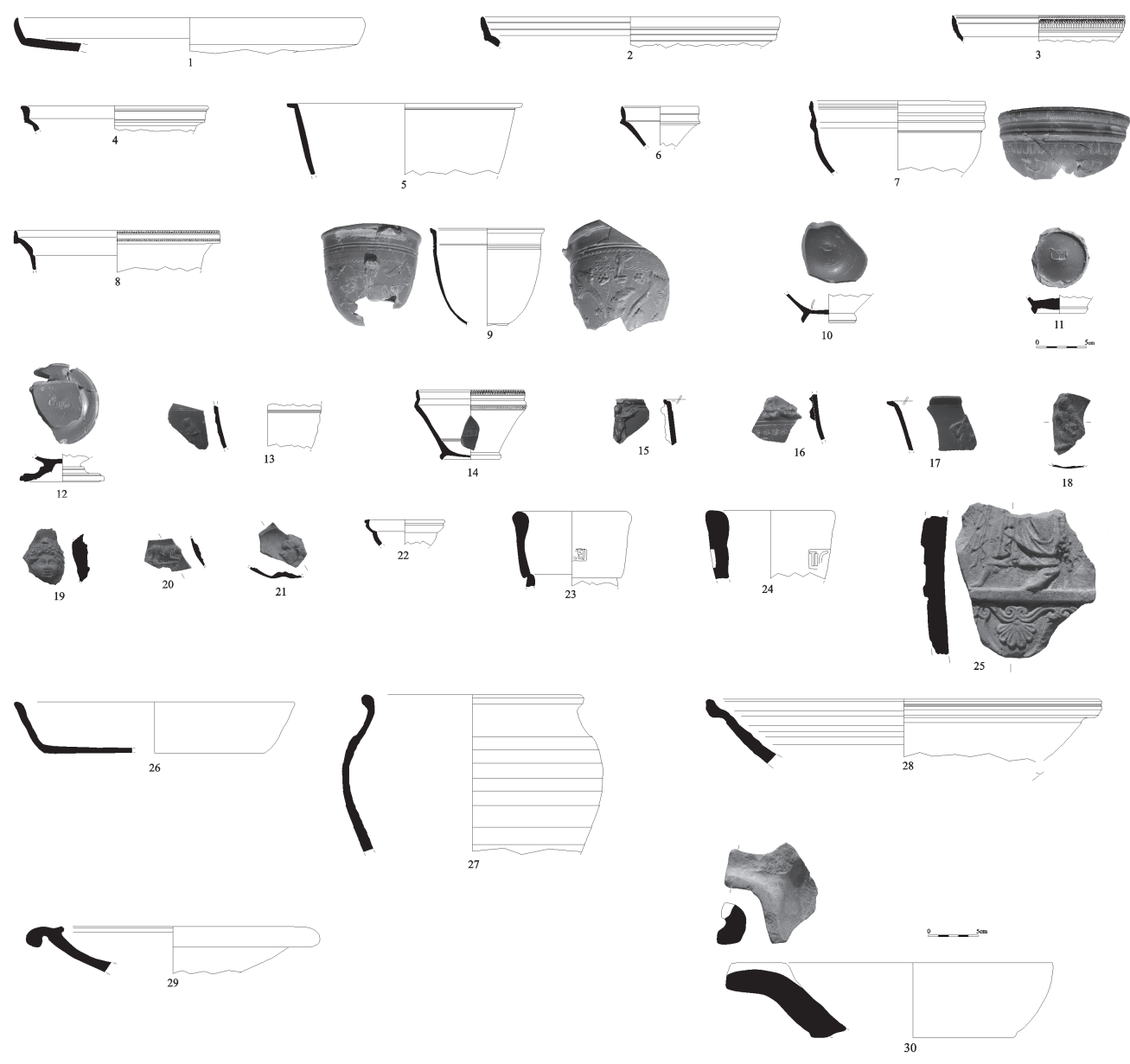

Figura 3. Una muestra de los materiales cerámicos recuperados en el mismo contexto en que se localizó el punzón del cérvido y que nos permite datar esta pieza en un momento situado entre los emperadores Tiberio y Claudio. 1: Presigillata Consp. 4. 2: TS sudgálica Drag. 15a. 3: TS itálica Consp. 31/33. 4: TS itálica forma indeterminada. 5: TS sudgálica forma indeterminada. 6: TS itálica Consp. 22.5. 7: TS itálica, cáliz, forma símil a Consp. R 99.2.2, con decoración de ovas y procesión de musas tocando sendas liras. 8: TS itálica R 2.1.1. 9: TS itálica, skyphos, forma VII de Dragendorff y Watzinger (1948), presenta decoración de guirnaldas, motivos vegetales, Apolo sobre pedestal, un sileno barbudo en el medallón del asa y sello rectangular (...)/ANNI atribuible al taller de los Annii (entre el 15 a. C. y el 15 d. C.). 10: TS itálica con sello rectangular PRIM. 11: TS itálica con sello rectangular CIELLV/P.CORN. 12: TS itálica con sello in planta pedis GELLI. 13: TS itálica con figura femenina. 14: TS itálica Consp. 22.1, con sello rectangular (...)MABR. 15: TS itálica cáliz con ninfa danzando. 16: TS itálica con lucha de serpiente y otro animal. 17: TS itálica con figura masculina desnuda y bastón. 18: Fragmento de disco de lucerna con pugilato. 19: Medallón de asa cerámico con cabeza de Gorgona. 20: Fragmento de disco de lucerna con león. 21: Fragmento de disco de lucerna con sátiro. 22: TS sudgálica Drag. 27b. 23: Ánfora tarraconense Pascual 1 con sello (...)CEl. 24: Ánfora tarraconense Pascual 1 con sello TIC(...). 25: Lastra Campana del tipo dos jóvenes flanqueando un candelabro. 26: Cazuela de cocina a imitación de la forma F6 Luni 5 de producción itálica. 27: Olla de cocina. 28: Lebrillo de cerámica común. 29: Mortero tipo Dramont D1. 30: Mortero de piedra local. 


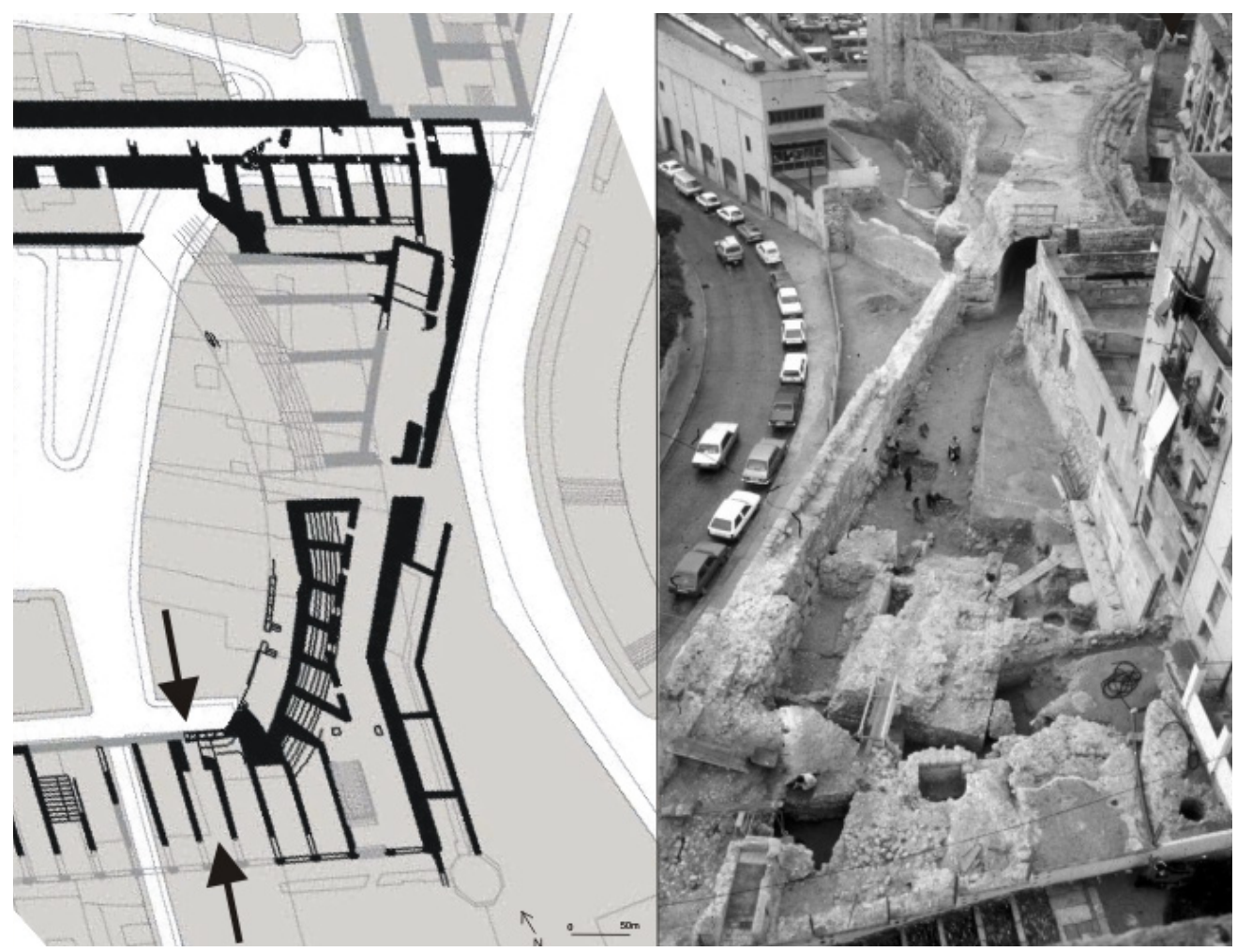

Figura 4. Izquierda, plano de la cabecera del circo a partir de Planimetria arqueològica de Tàrraco (Macias et al., 2007). Las flechas indican la situación de la bóveda K. Derecha, imagen general de las excavaciones del TED’A en la cabecera del circo, año 1989 (extraída de Mar et al., 2015: 171, fig. 116). Situación de la bóveda K en el extremo superior derecho de la imagen.

cronología moderna, dato que nos permite hipotetizar sobre el posible carácter residual de la pieza (Ajuntament de Tarragona - Arxiu TED'A).

\section{Análisis de los ejemplares tarraconenses}

\subsection{Sello con representación zoomorfa}

La primera pieza que vamos a analizar corresponde a un punzón hecho en cerámica con una pasta muy depurada de coloración beige y desgrasantes blanquecinos de granulometría fina (Cailleux M-85). Su estado de conservación es bueno, aunque se ha perdido aproximadamente la mitad del campo decorativo (fig. $5 a$ ). 
El punzón en sí tiene una cara de tendencia circular de unos 9 cm de diámetro y algo más de $1 \mathrm{~cm}$ de espesor. Presenta decoración a relieve, que serviría para plasmar el motivo en negativo en la masa panadera o bien para ser usado en un punzón matriz. La parte trasera, ligeramente espatulada, tendría una protuberancia de unos $4 \mathrm{~cm}$ de altura por 2,7 cm de grosor en la zona central, hecha a mano, como denotan las imperfecciones, así como las digitaciones. Esta parte permitiría aprehender la pieza y desmoldarla con facilidad de la masa. La pieza en sí tendría una altura de unos $5 \mathrm{~cm}$.

En relación a cómo se manufacturó, en primer lugar, se prediseñó el disco circular, posiblemente utilizando una plantilla matriz que le aportase esa morfología y ornato. Luego se le unió el mango trasero, que quedó fijo con barbotina y el cual, por medio de una espátula, terminó totalmente integrado con la parte delantera. Finalmente, la parte superior del pomo se concluyó modelándola a mano.

En cuanto al motivo decorativo, podemos decir que está dividido en dos registros concéntricos. El primero de ellos y más central, en el que aparece un cérvido mirando a la derecha y en movimiento. Se aprecia bien el prótomo, coronado por la cornamenta. La escena se desarrolla en un ambiente natural, como se desprende de la presencia de algunas ramas arbustivas que flanquean la figura central. Este medallón central, de unos $5 \mathrm{~cm}$ de diámetro, se encuentra flanqueado por una línea sogueada que daría paso a una cenefa que abrazaría este elemento central. Este último remate es una guirnalda corrida fitomorfa con pámpanos y hojas de vid. Para finalizar, de nuevo se repite la orla sogueada, que actúa a modo de gráfila de cierre.

Como ya hemos dicho previamente, la decoración está en positivo, algo que se aleja de la dinámica común de los ejemplares hasta el momento localizados, los cuales, en su mayoría, presentan los motivos incisos. Excepciones a este respecto, además del que ahora presentamos, son algunos sellos localizados en Elche con representación de pavo real (Salido y Bustamante, 2014: n. ${ }^{\circ} 45$ ), en Jumilla con una escena similar a la que ahora traemos a colación (Gijón y Bustamante, 2010: fig. 3) y en Oliva de Valencia con un bóvido (Salido y Bustamante, 2014: n. ${ }^{\circ} 70$ ), y que fueron interpretados como posibles "punzones madres» de sellos de panaderos (Salido y Bustamante, 2014: 52).

Un elemento altamente significativo en esta pieza es la presencia de un mango, una parte muy práctica si tenemos en cuenta que la plasticidad de la masa donde se iba a plasmar el motivo podría dificultar su desmolde y desvirtuar el motivo plasmado. Además, esta parte aportaría salubridad al producto al evitar el posible contacto de la masa con las manos. A pesar de estas — necesarias- funciones, son muy pocas las piezas que lo presentan. En concreto, tenemos el ejemplar de Alicante con pomo trasero a modo de protuberancia, pero con un desarrollo de unos $2,5 \mathrm{~cm}$ ligeramente inferior al que analizamos (Salido y Bustamante, 2014: n. ${ }^{\circ}$ 15). El otro ejemplar, procedente de Valencia, se articula a modo de pellizco practicado estando la pieza aún en fresco (Salido y Bustamante, 2014: n. $\left.{ }^{\circ} 70\right)$.

En relación a la iconografía de este tipo de piezas, es muy común la representación de figuras zoomorfas como los lemúridos de los sellos localizados en Alicante (Rubio, 

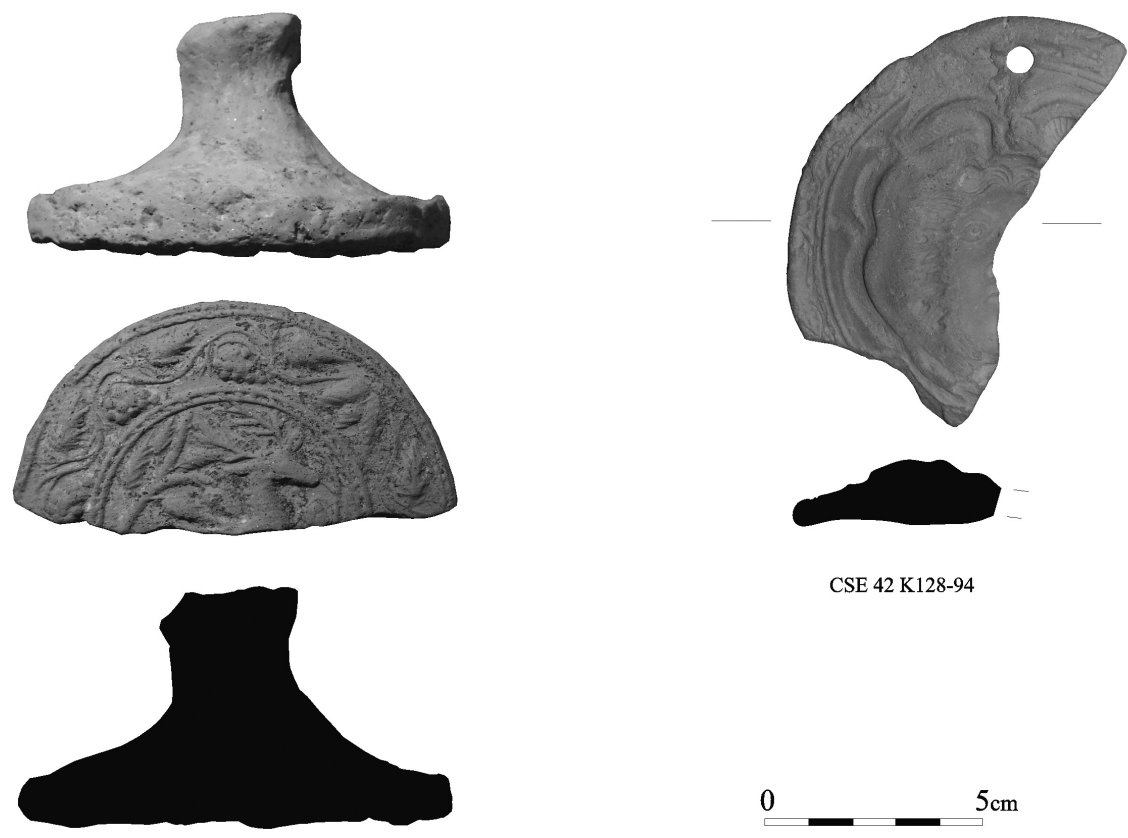

CSE 42 K128-94

TMC-07-1753-1700

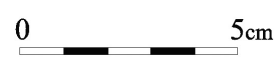

Figura 5. Piezas analizadas. Izquierda, punzón localizado en la excavación del Mercado Central (TMC-07-1753-1700). Derecha, disco localizado en el circo romano (CSE 42 K128-94) (Ajuntament de Tarragona - Arxiu TED’A).

1986: 311, fig. 125) o en Murcia (Medina, 2009: lám. 5), suidos como en Elche (Ramos, 1962: lám. LXXXV, n. ${ }^{\circ}$ 28), camélidos como en Lorca (Salido y Bustamante, 2014: n. ${ }^{\circ}$ 64), bóvidos como en Valencia (Salido y Bustamante, 2014: n. ${ }^{\circ}$ 67), aves del tipo pavo real como en el caso de Elche (Salido y Bustamante, 2014: n. ${ }^{\circ} 45$ ) o bucráneos de toros como en El Monastil (Poveda, 2012: fig. 24). Sin embargo, un estudio de compilación llevado a cabo por uno de los firmantes puso sobre la mesa que la representación de cérvidos, como el caso que ahora nos compete, es una de las más recurrentes y repetidas (Salido y Bustamante, 2014: fig. 32). Similares ejemplos los localizamos en el Cerro de los Santos (Serrano, 1987), Hellín (Poveda, 2012: fig. 24, n. ${ }^{\circ}$ 124), Cehegín (Lillo, 1981: n. ${ }^{\circ}$ 6), Jumilla (Gijón y Bustamante, 2010: fig. 3), Valencia con dos ejemplares (Salido y Bustamante, 2014: n. ${ }^{\circ} 68$ y 71) y Sagunto (Salido y Bustamante, 2014: 72). Lo mismo ocurre con la propia disposición de la cenefa exterior que envuelve la escena a modo de cenefa corrida con vides y pámpanos, con interesantes ejemplares en Alicante (Rubio, 1986: 311 , fig. 125). Además, el uso de estos frutos está bien atestiguado en los sellos localizados en Lucentum (Salido y Bustamante, 2014: n. ${ }^{\circ}$ 14), Elche (Salido y Bustamante, 2014: n. ${ }^{\circ} 45$ y 48), Murcia (Medina, 2009: lám. 5), Caravaca de la Cruz (Salido y Bustamante, 2014: n. ${ }^{\circ} 60$ ), Lorquí (Lillo, 1981: n. ${ }^{\circ}$ 5) y Valencia (Salido y Bustamante, 2014: n. ${ }^{\circ} 70$ ). 
La composición iconográfica presentada tiene un claro simbolismo asociable a los ciclos de regeneración, así como a la fecundidad. Concretamente, los cérvidos en época antigua aúnan una serie de aspectos que les conferían cierta sacralidad. Ciertamente, estos atributos radican en su cornamenta. En primer lugar, por la vigorosidad que les aporta y que supone su principal arma en la berrea, uno de los momentos cumbre de los ciclos reproductivos de esta especie. Y, en segundo lugar, en la regeneración de esos huesos por el proceso denominado «desmogue». Este acto natural y anual supone que los cérvidos se desprenden de sus cuernos e inician la generación de una nueva cornamenta. Por lo tanto, supone claramente el cierre y la abertura de un nuevo ciclo vital encaminado al momento de la berrea y, por consiguiente, buscando la procreación de la especie.

La presencia del cérvido en algunos pasajes mitológicos es amplia si nos remontamos a las narraciones homéricas. En concreto, nos referimos a su especial vinculación con Artemisa/Diana (LIMC, 1981-1984: s. v. "Artemis/Diana»). Entre los pasajes destaca aquel en el que Ovidio (Metamorfosis, III: 140-252) narra cómo Acteón, al ser descubierto admirando a Diana y sus ninfas en pleno baño, termina mutando en ciervo y es asesinado por sus propios perros. Además, las cornamentas, una vez desprendidas, también aparecen adornando algunos monumentos conmemorativos a esta divinidad, como en el caso de un bajo relieve localizado en la actualidad en los Museos Vaticanos (Daremberg y Saglio, 1982: s. v. "Diana», fig. 2374). En otras ocasiones, este animal aparece cortejándola o transportándola — como se percibe en las representaciones como Diana Lucífera en algunos denarios (RRC 207-1), o bien como atributo de la diosa en su variante efesia sujetando a un lado los cuernos de un ciervo y en la otra mano una lanza, como en los denarios emitidos por Lucio Hostilio Saserna (RRC 448-3)—. También la estatuaria mayor es bastante recurrente al presentar de manera continua a esta divinidad acompañada por este animal, siendo un caso ejemplificador la Diana de Versalles.

El culto a esta divinidad en la península ibérica tuvo mucho éxito, bien por un sustrato indígena que terminó calando en el ideario romano (Baena de Alcázar, 1989: 82) o bien como un guiño a algunos mandatarios que tuvieron cierta cercanía a las prácticas cinegéticas —en el caso de Itálica, la proliferación de sus estatuas apareció "como parte del mensaje político difundido por la remodelación urbanística general» que sufrieron determinadas ciudades, como apunta Oria (1999: 93).

En este contexto planteamos como una de las líneas de trabajo, a confirmar en un futuro, que este sello se haya usado para marcar panes que se ingirieron en alguna festividad en la que el cérvido hubiera tenido un papel activo. Sugerimos como hipótesis una vinculación con la diosa Diana, a la que nos hemos referido en párrafos previos. A este respecto nos sirve de ejemplo una festividad, el día 13 de agosto, que llevaba por nombre Nemoralia y que, mayoritariamente, se festejaba en el centro de Italia. El núcleo geográfico de máxima expresión era el entorno del lago Nemi, donde se encontraba un templo dedicado a esta divinidad. Durante la celebración de la festividad, además de danzas, procesiones, quema de antorchas y comidas campestres, se llevaban a cabo ofrendas, algunas de ellas 
comestibles, como panecillos con formas (en los que se usarían moldes y sellos como el que ahora presentamos), algunos representando partes enfermas del cuerpo, y con los que se pedía curación (Wissowa, 1912: 198-203; Alföldi, 1960: 137-144; Maassa-Pairault, 1969: 425-471; Blagg, 1986: 21 1-220; Green, 2007). Aunque esta festividad no está atestiguada por el momento en el espacio geográfico al que ahora nos referimos, podría ser sugerente y ejemplificadora para futuros análisis.

\subsection{Sello con Medusa}

La otra pieza localizada también corresponde a un disco cerámico (fig. 5b), de similares dimensiones: ca. $9 \mathrm{~cm}$ de diámetro, de los que en su lado más desarrollado se conservan unos $7 \mathrm{~cm}$. Presenta un grosor medio de unos 0,8-1 cm, aunque en determinados puntos una protuberancia alcanza hasta los $1,5 \mathrm{~cm}$. Está hecho en cerámica muy depurada de coloración marronácea (Cailleux L-70). Resulta de interés el orificio practicado durante la precocción en la zona superior de la pieza, de cuya función luego aportaremos una posible hipótesis de trabajo.

A diferencia de la pieza anterior, ésta no presenta mango trasero. En su cara delantera tiene una representación en negativo, mientras que en la trasera presenta alisado hecho a mano, como se desprende de las digitaciones del figulus. La zona que se imprimía en la masa tiene una representación central que ocupa todo el campo decorativo. Aunque parcialmente obliterada, se percibe la representación de un busto de la Medusa de tipo helenístico y romano, es decir, con rasgos apacibles y bellos. La figura aparece centralizada, con los ojos abiertos y rictus serio. Su frente aparece culminada por un flequillo doble, coronado por dos alas decoradas por medio de incisiones que vienen a representar plumas esquemáticas. En el centro de las alas aparece una protuberancia circular, desde la que salen dos líneas incisas que van a flanquear la figura hasta el cuello y que son claramente la representación esquemática de serpientes que aderezarían la cabellera de la Medusa y que fueron uno de sus atributos más recurrentes. En la parte de la barbilla se atisba la presencia de algún elemento que se ha perdido, posiblemente las serpientes anudadas, un rasgo característico de esta figura.

Toda la imagen se encuentra delimitada por una doble línea excisa que actuaría de límite de la representación y que, además, se acompaña por una estrecha cenefa de pámpanos que se ha borrado y que es casi imperceptible en la actualidad. Un ejemplo con similar margo sería el localizado en la Albufereta (Salido y Bustamante, 2014: n. ${ }^{\circ} 1$ ).

Hasta el momento no se han localizado paralelos iconográficos de esta pieza en la península ibérica, en lo que se refiere a los sellos alimentarios. En este sentido, este ejemplo guarda parecido con el emblema central del mosaico de la Medusa, procedente de la zona residencial de la ciudad ${ }^{1}$. En este caso, de nuevo la imagen presenta los mismos atributos,

1. Se recuperó durante las obras asociadas a la explotación de la cantera del puerto moderno. Desde el año 1857 está depositado en el Museo Nacional Arqueológico de la ciudad, con el número de inventario MNAT 2921. 
así como facciones, y se enmarca en un medallón circular. Esta pieza ha sido datada en época severiana (finales del siglo II d. C. - primer tercio del III d. C.) y se ha atribuido a una officina del Egeo (Navarro, 1979: 175-180).

Además, no hay que olvidar los clípeos del foro de Tarraco donde aparece Medusa junto con otras representaciones (Macias et al., 2007: 763-787; 2011: 877-886; Mar et al., 2015: 83-129; o, más recientemente, Peña, 2018: fig. 4).

En relación a cuándo se usaría este tipo de piezas, nos parece sugestiva su posible asociación con Minerva, quien muy recurrentemente usa en algunos de sus atributos - como escudos o coraza - la efigie de Medusa. En Tarraco se ha evidenciado una gran concentración, diversidad y tipos de culto relacionados con Minerva, no sólo desde el punto de vista privado (exvotos, pavimento musivo e inscripciones) o semipúblico (asociaciones militares o artesanales como la schola del collegium fabrum) (Koppel, 1988), sino también público (existieron, al menos, dos templos consagrados a la diosa: el templo capitolino, situado en el foro de la colonia, y el de Minerva Augusta, hoy aún sin ubicación precisa) (Ruiz, 2017: 341-344), y por lo tanto con su vinculación con el poder estatal y los clípeos del complejo forense ${ }^{2}$.

Esta última idea enlaza con la posible función que otorgamos a este sello vinculada con actos públicos, sugerencia que se cimenta en la escasa presencia de este tipo de sellos en el registro arqueológico, lo cual apuntaría a una práctica puntual. Creemos que estas piezas sellarían panes destinados a la ingesta en festividades públicas o conmemorativas, siendo el circo un lugar ideal para dichos repartos.

Los sellos alimentarios con iconografía alusiva al poder imperial son abundantes en la península ibérica. Ejemplos de ello se han localizado en Benalmádena (Rodríguez, 1982), en los que se representa un águila con las alas desplegadas portando una corona, o bien el de Cabecico del Tesoro en Verdolay (Lillo, 1981: n. ${ }^{\circ}$ 1) y el de Monte Aralí en Murcia (Blázquez, 1986), ambos con representación de la loba capitolina con los dos niños.

Y, por último, no podemos dejar de apuntar dos evidencias más que apuntan a su función como sello de masas panaderas. La primera, que en el extremo opuesto donde se recuperó este sello, se halla un conjunto de espacios abovedados interpretados como horreum (Dupré et al., 1988; Dupré y Subías, 1993: 603-609; Piñol, 2000: 89-107; Macias et al., 2007a: 767-771; Mar et al., 2012: 341; Vinci et al., 2014: 1-20). Y la segunda, la recuperación en una zona cercana (a escasos 100 metros de la cabecera del circo, y de nuevo durante la explotación de la cantera del puerto moderno) de un catillus de molino de tracción animal, con inscripción (RIT $814=$ CIL II2/14, 1892) e interpretada como $R$ (ota) Boaeti (Rodà, 2002; Gorostidi et al., 2019: 235-242) ${ }^{3}$.

2. Debemos recordar, también, el relieve con inscripción situado en la torre homónima de la primera fase de la muralla republicana. Una representación en honor a Minerva que la acredita como la custos urbis, la protectora de la Urbs, en la ciudad (Pina, 2013: 111-119; Ruiz, 2017: 337-338).

3. Número de inventario MNAT 2505. Sus medidas: $65 \mathrm{~cm}$ de altura por $75 \mathrm{~cm}$ de diámetro. En el momento de su hallazgo (27 de agosto de 1863) se recuperó también la meta, la cual se rompió durante su posterior traslado. 


\section{Conclusiones}

Se han presentado dos piezas que suponen una ampliación de la nómina de sellos de panaderos hasta el momento conocidos. Están realizadas en barro, con una morfología idéntica a los tradicionalmente denominados "sellos de panaderos». En relación a la procedencia de estas piezas, a falta de estudios arqueométricos, apostamos por una procedencia localregional que se adapta a la idiosincrasia religiosa. Esta idea se complementa con el hecho de que la pieza con la representación de cérvido fue hallada en un ambiente figlinario (Roig, 2014) y, por consiguiente, podría ser una producción local que terminó siendo desechada por algún problema funcional de la misma.

Además de la aportación de un elemento iconográfico inédito para estos sellos en la península Ibérica como es la Medusa, son dos los datos de interés para ampliar el conocimiento que tenemos de este fenómeno en Hispania.

En primer lugar, la contextualización cronológica de una de las piezas en pleno siglo I d. C., la cual, junto con el dato aportado para Lucentum (Salido y Bustamante, 2014: 54), que apunta al siglo II, o el de Malaca, inserto entre el II-IV d. C. (Pérez-Malumbres, 2018: 85), son prácticamente los únicos datos con los que contamos para datar este fenómeno.

En segundo lugar, la contextualización espacial en ambos casos. La primera pieza, en un contexto claramente productivo, asociado a estructuras artesanales; y la segunda, en un edificio lúdico: el circo. Aunque a día de hoy no se ha localizado ninguna instalación alimentaria en el circo de Tarragona, sí nos parece sugerente su localización en un espacio público, sobre todo teniendo en cuenta la escasa presencia de los sellos en estos contextos, hecho que induce a pensar en un sellado no doméstico cotidiano, sino puntual y asociado a festividades concretas.

La ingesta de panecillos y pasteles con formas múltiples fue ampliamente recogida por las fuentes clásicas, no sólo en ambientes privados, como en la casa de Trimalción (Petronio, Satyr., 60: 4-5); sino también en escenas vinculadas a los emperadores, como en el referido por Suetonio en relación al emperador Domiciano (Suetonio, Domic., VIII: 4 y 6), o de manera más directa en edificios de espectáculos, como la alusión hecha a las popinae de los teatros (Marcial, Epigram., XI: 31). Dentro de este estudio cobra especial significación este último pasaje, ya que uno de los ejemplares localizados también procede de un espacio lúdico. Esto no es un hecho aislado, puesto que para Hispania se han localizado dos ejemplares de moldes para pasteles en edificios de espectáculos; en concreto, uno para el teatro de Córdoba (Ventura, 2002) y otro para el entorno del teatro de Cádiz (Salido y Bustamante, 2014: n. ${ }^{\circ}$ 6). Esto hablaría de una práctica ya esbozada por las fuentes clásicas y que esperamos que en un futuro próximo se pueda confirmar en el espacio que ahora analizamos.

En relación a la iconografía, ésta es altamente simbólica, lo que apoyaría la idea de que estamos ante objetos que tienden a plasmarse en alimentos con una significación religiosa. En cuanto al cérvido, podríamos estar ante la representación de un símbolo asociado a Diana. Planteamos, por lo tanto, como hipótesis, su ingesta en algunas festividades sig- 
nificativas para su culto. Aunque no está atestiguada para Tarraco, tenemos en mente la Nemoralia, donde el consumo de panecillos con formas específicas puede ser sugerente a este respecto.

La segunda, con representación de Medusa, alude a un símbolo muy recurrente en la ciudad, presente en algunos de los edificios más significativos de la capital, y que vincularíamos a posibles repartos que se podrían haber desarrollado en ámbito lúdico, como en el circo.

Hasta el momento, los mapas de distribución de estas piezas en ámbito peninsular se concentran de manera muy abigarrada en la zona del sureste peninsular, con escasa presencia en ámbito lusitano. Su existencia en ámbito tarraconense nos muestra un arco geográfico que, hasta el momento, no presentaba piezas en estos elencos, ya que los más cercanos al lugar se localizaban en Valencia y Sagunto (Salido y Bustamante, 2014: n. ${ }^{\circ}$ 67-72).

El único estudio a nivel peninsular de compendio pone sobre la mesa los tipos iconográficos más reproducidos, entre los que se encuentran las divinidades asociables a ciclos agrícolas, las del mundo militar o bien del culto imperial, escenas teatrales, mitológicas o consumaciones matrimoniales (Salido y Bustamante, 2014: 53). Los dos casos planteados se insertan plenamente en algunos de dichos discursos. A todo ello se asocia una morfología similar a los otros sellos peninsulares que permite apuntar a esta hipótesis de trabajo.

\section{Agradecimientos}

Quisiéramos agradecer a Joan Menchon (MHT) y a Josep Anton Remolà (MNAT) todas las facilidades y su total predisposición para llevar a cabo el presente artículo. Agradecemos también a Marta Nicolás (becaria predoctoral FI Generalitat de Catalunya URV-ICAC) y a Jesús Carruesco (URV-ICAC) sus consejos a nivel bibliográfico sobre la Nemoralia y el culto a Diana.

\section{Fuentes}

MARCIAL, Epigramas, II (Traducción y notas de J. Fernández y A. Ramírez), Editorial Gredos, 2001.

OVIDIO, Metamorfosis, Libros I-V (Introducción, traducción y notas de J. C. Fernández y J. Cantó), Editorial Gredos, 365, 2008.
PETRONIO, El Satiricón (Introducción, traducción y notas de L. Rubio), Editorial Gredos, 1978.

SUETONIO, Vida de los doce césares (Traducción y notas de R. M. ${ }^{a}$ Agudo), Editorial Gredos, 168, 1992. 


\section{Bibliografía}

ALFÖLDI, A., 1938, Tonmodel und Relifmedaillons aus den Donauländern, Dissertationes Panonicae, Laureae Aquincenses I, Leipzig.

ALFÖLDI, A., 1960, Diana Nemorensis, American Journal of Archaeology 64/2, 137-144.

ALFÖLDY, G., 1975, Die römische Inschriften von Tarraco, Madrider Forschungen 10, Walter De Gruyter \& Co., Berlín.

ALFÖLDY, G., 1991, Tarraco, Fòrum. Temes d'història i d'arqueologia tarragonines 8, MNAT, Tarragona.

ALFÖLDY, G., 1992, Tarraco y la Hispania romana: cultos y sociedad, en M. MAYER y J. GÓMEZ (eds.), Religio deorum. Actas del Coloquio Internacional de Epigrafía "Culto y sociedad en Occidente», Ed. Ausa, Sabadell, 7-26.

BAENA DE ALCÁZAR, L., 1989, La iconografía de Diana en Hispania, Boletín del Seminario de Estudios de Arte y Arqueología 55, 79-1 12.

BESIG, H., 1937, Gorgo und Gorgoneion in der archaischen griechischen Kunst, Berlín.

BLAGG, T. F. C., 1986, The cult and sanctuary of Diana Nemorensis, en M. HENIG y A. KING (eds.), Pagan Gods and Shrines of the Roman Empire, Oxford University Press, Oxford, 211-220.

BLÁZQUEZ, J. M., 1960, Molde de barro con el tema de la loba y los gemelos, Zephyrus 11, 258-259.

BRÚ, M., GARCIA, M., ROIG, J. F. y TEIXELL, I., 2012, Noves dades sobre les àrees residencials de la ciutat de Tàrraco, Tribuna d'Arqueologia 20102011, 313-335.

BUSTAMANTE, M., SALIDO, J. y GIJÓN, E. 2014, La panificación en la Hispania romana, en M. BUSTAMANTE y D. BERNAL (coords.), Artífices idóneos: artesanos, talleres y manufacturas en Hispania, Anejos de Archivo Español de Arqueología LXV, 333-369.

CARPENTER, T. H., 1991, Art and Myth in Ancient Greece, Londres.
CHÁVARRI, O., 2011, El culto de Minerva en Hispania: custos urbis de Roma y las provincias, en T. NOGALES e I. RODÀ (eds.), Roma y las provincias: modelo y difusión, XI Coloquio Internacional de Arte Romano Provincial (Mérida 2009), Hispania Antigua, Serie Arqueológica 3, Roma, 1025-1029.

CIL II2/14 = Corpus Inscriptionum Latinarum, II. Inscriptiones Hispaniae Latinae, Editio Altera, Pars XIV, Conventus Tarraconensis.

DAREMBERG, C. y SAGLIO, E., 1892, Dictionnaire des antiquités grecques et romaines d'après les textes et les monuments, París.

DE LA TORRE, J., 1921, Hallazgos arqueológicos junto a Córdoba, Boletín de la Real Academia de la Historia 79, 419-421.

DE LOS SANTOS, S., 1949, Moldes de barro romano, Actas y memorias de la Sociedad Española de Antropología, Etnografía y Prehistoria 24, 1-4, 62-82.

DEONNA, W., 1919, Notes d'archéologie suisse. Décoration murale de Corsier, Anzeiger für Schweizerische Altertunskunde, 85-96.

DÍAZ, M. y TEIXELL, I., 2017, Noves dades del circ romà de Tàrraco: les darreres excavacions al carrer Trinquet Vell i a l'àrea de la porta triomfal, Tribuna d'Arqueologia 2014-2015, 217-238.

DRAGENDORFF, H. y WATZINGER, C., 1948, Arretinische Reliefkeramik, Reutlingen.

DREXEL, F., 1916, Crustulum et mulsum, Römisch-germanisches Korrespondenzblatt 9, 17-22.

DUPRÉ, X., MASSÓ, J., PALANQUES, M. Ll. y VERDUCHI, P. A., 1988, El Circ romà de Tarragona. I. Les voltes de Sant Ermenegild, Excavacions Arqueològiques a Catalunya 8, Barcelona.

DUPRÉ, X. y SUBÍAS, E., 1993, Els precedents de l'anomenat Pretori de Tarragona, Homenatge a Miquel Tarradell, Estudis Universitaris Catalans XXIX, Barcelona, 603-609.

ETTLINGER, E., HEDINGER, B., HOFFMANN, B., KENRICK, P. M., PUCCI, G., ROTH-RUBI, K., 
SCHNEIDER, G., VON SCHNURBEIN, S., WELLS, C. M. y ZABEHLICKY-SCHEFFENEGGER, S., 1990, Conspectus Formarum Terrae Sigillatae Italico Modo Confectae, Römisch-Germanische Kommission des Deutschen Archäologischen Instituts zu Frankfurt a. M., Bonn.

FLOREN, J., 1977, Studien zur Typologie des Gorgoneion, Münster/Westfalia.

GIJÓN, M. E. y BUSTAMANTE, M., 2010, Los sellos romanos de panadero: una aproximación a su estudio a partir de los depositados en el Museo Nacional de Arte Romano (Mérida), Huelva en su historia 13, 15-30.

GOROSTIDI, D., LÓPEZ, J. y ROYO, H., 2019 , Inscribed Pompeian millstones from Tarragona, Spain, en T. J. ANDERSON y N. ALONSO (eds.), Tilting at Mills. The Archeology and Geology of Mills and Milling. Proceedings of the Colloquium Held at the Museum of Archaeology of Almería, 2014, Revista d'Arqueologia de Ponent 19, extra 4, 235-244.

GREEN, C. M. C., 2007, Roman Religion and the Cult of Diana at Aricia, Cambridge University Press, Nueva York.

HOWE, T. P., 1954, The Origin and Function of the Gorgon-Head, American Journal of Archaeology LVIII/3, 209-221.

HUGHES, S. L. y FERNANDEZ, J. A., 1981, Las Gorgonas: guardianas de lo sagrado, Argos V, 53-73.

KOPPEL, E. M., 1988, La Schola del collegium fabrum de Tarraco y su decoración escultórica, Faventia Monografies 7, UAB, Barcelona.

LILLO, P. A., 1981, Contribución al estudio de los sellos de panadero del Sureste, Memorias de Historia Antigua 5, 187-194.

LIMC, 1981-1984, Lexicon Iconographicum Mythologiae Classicae, "Artemis» y "Artemis/ Diana», Vol. II, Zúrich-Múnich.

MAASSA-PAIRAULT, F. H., 1969, Diana Nemorensis, déesse latine, déesse hellénisée, Mélanges d'archéologie et d'histoire 81/2, 425-471.

MACIAS, J. M., FIZ, I., PIÑOL, Ll., MIRÓ, M. T. y GUITART, J., 2007, Planimetria arqueològica de Tàrraco, Documenta 5, ICAC, Tarragona.
MACIAS, J. M., MENCHON, J., MUÑOZ, A. Y TEIXELL, I., 2006, Intervencions arqueològiques a la catedral de Tarragona: treballs del Pla Director (2000-2002), Tribuna d'Arqueologia 2003-2004, 221-246.

MACIAS, J. M., MENCHON, J., MUÑOZ, A. Y TEIXELL, I., 2007a, Excavaciones en la catedral de Tarragona y su entorno: avances y retrocesos en la investigación sobre el culto imperial, en T. NOGALES y J. GONZÁLEZ (eds.), Actas del Congreso Internacional «Culto Imperial: politica y poder» (Mérida, 2006), L’Erma di Bretschneider, Roma, 764-787.

MACIAS, J. M., MENCHON, J., MUÑOZ, A. y TEIXELL, I., 2007b, L'Arqueologia de la Catedral de Tarragona. La memòria de les pedres, $L a$ Catedral de Tarragona. In Sede, 10 anys del Pla Director de Restauració, Tarragona, 151-213.

MACIAS, J. M., MENCHON, J., MUÑOZ, A. y TEIXELL, I., 2010, La construcción del recinto imperial de Tarraco (provincia Hispania Citerior), en J. LÓPEZ y O. MARTIN (eds.), Tarraco: construcció $i$ arquitectura d'una capital provincial romana, Actes del Congrés Internacional en Homenatge a Th. Hauschild (Tarragona, 2009), Butlletí Arqueològic V/32, 423-479.

MACIAS, J. M., MUÑOZ, A., PEÑA, A., RAMON, M. y TEIXELL, I., 2012, Praesidium, Templum et Ecclesia. Les intervencions arqueològiques a la Catedral de Tarragona (2010-2011). Memòria d'una exposició temporal, Tarragona.

MACIAS, J. M., MUÑOZ, A., TEIXELL, I. y MENCHON, J., 2011, Nuevos elementos escultóricos del recinto de culto del Concilium Provinciae Hispaniae Citerioris (Tarraco, Hispania Citerior), en T. NOGALES e I. RODÀ (eds.), Roma y las provincias: modelo y difusión, XI Coloquio Internacional de Arte Romano Provincial (Mérida 2009), Hispania Antigua, Serie Arqueológica 3, Roma, 873-886.

MAR, R., RUIZ DE ARBULO, J., VIVÓ, D. Y BELTRÁN-CABALLERO, J. A., 2012, Tarraco. Arquitectura y urbanismo de una capital provincial romana. Vol. I. De la Tarragona ibérica a la construcción del templo de Augusto, Documents d'Arqueologia Clàssica 5, Tarragona. 
MAR, R., RUIZ DE ARBULO, J., VIVÓ, D., BELTRÁN-CABALLERO, J. A. y GRIS, F., 2015, Tarraco. Arquitectura y urbanismo de una capital provincial romana. Vol. II. La ciudad imperial, Documents d'Arqueologia Clàssica 6, Tarragona.

MEDINA, A. J., 2009, Excavación arqueológica de un ámbito urbano en época romana, la plaza de la Iglesia de Monteagudo (Murcia), Memorias de Arqueología de la Región de Murcia (2002-2003) 15. 191-216.

MOREAU, A., 1986, La race de Méduse : forces de vie contre forces de mort, Mort et fécondité dans les Mythologies, París.

MÜLLER, A., 1909, Die Neujahrsfeier im römischen Kaiserreiche, Philologus LXVIII, 4, 464-487.

NAVARRO, R., 1979, Los mosaicos romanos de Tarragona, Tesis doctoral inédita, Universitat de Barcelona.

ORIA, M., 1999, Diana en Itálica: una hipótesis, Faventia 21/2, 85-93.

PASQUI, A., 1906, Ostia. Nuove scoperte presso il Casone, Notizie degli Scavi Antique, 357-373.

PEÑA, A., 2018, El pórtico del recinto de culto a Divus Augustus en la acrópolis de Tarraco: la decoración del ático y su reflejo en las ciudades romanas de la Galia, Zephyrus, LXXXII, juliodiciembre 2018, 167-185.

PÉREZ-MALUMBRES, A., 2018, Sello cerámico de panadero de época romana con representación de dextrarum iunctio, procedente de Málaga, Albahri entre oriente y occidente. Revista independiente de estudios históricos 4, 83-98.

PINA, F., 2013, Minerva, custos urbis de Roma y de Tarraco, Archivo Español de Arqueología 76, 111 119.

PIÑOL, Ll., 2000, Voltes del Pretori (C/ Enrajolat i Casa dels Militars), en R. CORTÉS y O. CURULLA (eds.), Intervencions arqueologiques a Tarragona $i$ entorn (1993-1999), Universitat Rovira i Virgili, Tarragona, 89-107.

POVEDA, A., 2012, La producción de lucernas en el sureste peninsular: primeros datos, en
D. BERNAL y A. RIBERA (eds.), Cerámicas hispanorromanas II. Producciones regionales, Cádiz, 353-369.

QUINTERO, P., 1942, Moldes y matrices de barro procedentes de Tamuda, Revista Mauritana de Tánger 172.

RAMOS, A., 1962, Excavaciones en La Alcudia: memoria de las practicadas durante 1953 , Noticiario Arqueológico Hispánico 5, 91-97.

RODÀ, I., (com.), 2002, Un recorregut per l'exposició Scripta Manent, en R. COMES e I. RODÀ (eds.), Scripta manent: la memoria escrita de los romanos, Catálogo de la exposición, Barcelona, 26-51.

RODRÍGUEZ, P., 1982, La arqueología romana de Benalmádena, Ayuntamiento de Benalmádena, Málaga.

ROIG, J. F., 2014, La figlina del Mercat Central: evidències d'un taller ceràmic al suburbi occidental de la colònia Tàrraco, Revista d'Arqueologia de Ponent 24, 55-74.

ROIG, J. F. y TEIXELL, I., 2015, Una sala de jocs als afores de Tàrraco: l'aprofitament d'espais de treball com a lloc de serveis, August $i$ les provincies occidentals, $2 n$ Congrés Internacional d'Arqueologia $i$ Món Antic (Tarragona, 2014), Tarragona, 221-227.

RUBIO, F., 1986, La necrópolis ibérica de la Albufereta de Alicante (Valencia, España), Serie Arqueológica 11, Academia de Cultura Valenciana, Valencia.

RUIZ, J. C., 2017, El culto a Minerva en Tarraco, Espacio, Tiempo y Forma 30, Serie II, 323-350.

SALIDO, J. y BUSTAMANTE, M., 2014, Pistrina Hispaniae. Panaderías, molinerías y el artesanado alimentario en la Hispania Romana, Monographies Instrumentum 47, Éditions Monique Mergoil, Montpellier.

SÁNCHEZ DE PRADO, M. ${ }^{a}$ D., 2004, El vidrio romano en el Conventus Carthaginensis, en A. FUENTES DOMÍNGUEZ (ed.), Jornadas sobre el vidrio en la España romana (La Granja, 2001), Fundación Centro Nacional del Vidrio, 83-85. 
SERRANO, D., 1987, Sello de panadero del Cerro de los Santos (Montealegre del Castillo, Albacete), Al-Basit 13/21, 65-68.

TEIXELL, I., 2012, Nous contextos arqueològics relacionats amb l'entrada dels francs a Tàrraco en el segle III dC. La moneda com a element de datació post quem per a la recuperació de la ciutat, en M. CAMPO (coord.), La moneda en temps de crisi. XVI Curs d'història monetària d'Hispània, Barcelona, 87-103.

TEIXELL, I., ROIG, J. F. y GOROSTIDI, D., 2016, El santuari romà de les nimfes del carrer Cristòfor Colom de Tarragona, Butlletí Arqueològic V/3435, 115-131.

VENTURA, Á., 2002, Kuchenform (molde de cocina) con escena teatral, en Á. VENTURA,
C. MÁRQUEZ, A. MONTERROSO y M. A. CARMONA (eds.), El teatro romano de Córdoba, 231-235.

VERNANT, J.-P., 1985, La mort dans les yeux, París.

VINCI, M. S., MACIAS, J. M., PUCHE, J. M., SOLÀ-MORALES, P. y TOLDRÀ, J. M., 2014, El subsuelo de la Torre del Pretorio: substructiones de tradición helenística bajo la sede del Concilium Prouinciae Hispaniae Citerioris (Tarraco), Arqueología de la Arquitectura 11, 1-20.

VV. AA., 2009, Tarraco, pedra a pedra. Catàleg de l'exposició, Tarragona.

WISSOWA, G., 1912, Religion und Kultus der Römer, Handbuch der klassischen AltertumsWissenschaft V, Múnich. 